\title{
Indicadores de sustentabilidad en empresas manufactureras y vinculación con instituciones generadoras de conocimiento: estudio de caso *
}

\author{
Sustainability Indicators in Manufacturing Companies and Links with \\ Knowledge-Generating Institutions: A Case Study
}

\author{
Ana Esther Escalante-Ferrer \\ Doctora en Educación, Universidad Autónoma del Estado de Morelos, \\ Cuernavaca -México, ana.escalante@uaem.mx \\ María del Carmen Torres-Salazar \\ Doctora en Logística y Dirección de la Cadena de Suministro, \\ Universidad Autónoma del Estado de Morelos, \\ Cuernavaca -México, maria.torres@uaem.mx \\ Alejandro García-Garnica \\ Doctor en Estudios Organizacionales, Universidad Autónoma del Estado de Morelos, \\ Cuernavaca -México, alejandro.garciag@uaem.edu.mx
}

Cómo citar / How to cite

Escalante Ferrer, A. E.; Torres-Salazar, M. del C.; García-Garnica, A. (2020). Indicadores de sustentabilidad en empresas manufactureras y vinculación con instituciones generadoras de conocimiento: estudio de caso. Revista CEA, v. 6, n. 12, 29-46. https://doi.org/10.22430/24223182.1601

Recibido: 25 de febrero de 2020

Aceptado: 30 de marzo de 2020

\section{Resumen}

La relevancia del artículo consiste en mostrar la relación entre instituciones generadoras de conocimiento y empresas manufactureras medianas y grandes del estado de Morelos, México. El propósito es analizar la correlación de los indicadores de sustentabilidad en sus cadenas de suministro y la vinculación que mantienen con universidades o centros de investigación. Se utilizó una metodología cuantitativa por encuesta, con resultados positivos de validez y confiabilidad del

\footnotetext{
* Este artículo se deriva del proyecto titulado «Relación entre indicadores de sustentabilidad en las cadenas de suministro y vinculación académica» y ha sido financiado con recursos otorgados por el Programa para el Desarrollo Profesional Docente Prodep, Apoyo a la Reincorporación de Exbecarios, Carta de liberación DSA/103.5/16/10404 Folio UAEMOR-EXB115.
} 
instrumento. Los resultados arrojan que, aun cuando existan acuerdos formales entre empresas e instituciones generadoras de conocimiento, no se muestran cambios en las prácticas de sustentabilidad. Pese al potencial de líneas de generación y aplicación innovadora de conocimientos, la amplia infraestructura y las bases de datos de las instituciones, el panorama general del nivel de implementación de los indicadores de sustentabilidad en las empresas que se obtuvo, es posible afirmar que la correlación estadística de la vinculación entre empresas e instituciones va de mediana a muy débil. Lo importante es crear las condiciones para que en el corto y mediano plazo se fortalezcan las relaciones entre las empresas, las instituciones y el Gobierno.

Palabras clave: indicadores, sustentabilidad, industria de transformación, vinculación, estudio de caso.

Clasificación JEL: L69; D89.

\begin{abstract}
The aim of this paper is to show the relationship between knowledge-generating institutions and medium- and large-sized manufacturing companies in the state of Morelos, Mexico. For this purpose, we analyze the correlation between the sustainability indicators implemented across the companies' supply chains and their links with universities or research centers. To collect the data, we used a quantitative questionnaire-based approach which proved to be a valid and reliable measurement tool. The results show no changes in sustainability practices even when there are formal collaborative agreements between companies and knowledge-generating institutions. Despite the potential of the lines of knowledge generation and innovative application of these latter and their extensive infrastructure and databases, we may conclude that the statistical correlation between the level of implementation of sustainability indicators in the companies under study and their linkage with knowledge-generating institutions ranges from medium to very weak. Therefore, the necessary conditions should be created so that the relationships between companies, such institutions, and the government are strengthened in the short and medium term.
\end{abstract}

Keywords: Indicators, sustainability, transformation industry, academic linkage, case study.

JEL classification: L69; D89.

\title{
1. INTRODUCCIÓN
}

Este artículo analiza la relación entre instituciones generadoras de conocimiento (IGC) y empresas, a partir de considerar algunos indicadores de sustentabilidad ligadas a las cadenas de suministro que estas últimas tienen. Ambas instancias requieren adecuarse oportunamente a las demandas del contexto en que se desenvuelven. El texto se presenta de manera deductiva. Se parte de los antecedentes y conceptos relacionados con la temática de investigación para posteriormente ofrecer una problematización que muestra la importancia de estudiar el tema de la vinculación. Más adelante se puntualizan los objetivos y la hipótesis de trabajo. Posteriormente, se presentan la metodología y los resultados del análisis estadístico, y al final, la discusión y las conclusiones correspondientes. Estos resultados indican la necesidad de estrechar la colaboración entre IGC y empresa y aprovechar el potencial que ofrecen las instituciones educativas y de investigación a la sociedad en su conjunto a fin de aspirar a un modelo económico social más sustentable. 
Durante los últimos años se ha generado una preocupación creciente, tanto por los gobiernos como por los investigadores, de repensar el modelo económico de las naciones en términos no solamente de crecimiento y desarrollo, sino además de lograr la sustentabilidad (Fuster Morell \& Espelt, 2019). Este último concepto es más amplio y alude, en términos generales, al logro de objetivos de desempeño económico vinculados a un uso adecuado de los recursos naturales en los procesos productivos, a la utilización de energías limpias y de tecnologías con bajo impacto ambiental, pero también alude a la equidad social de las actuales y futuras generaciones (Gutiérrez et al., 2019; Schrand, 2020). Hay algunas evidencias de que tanto las empresas como las universidades están preocupadas por ser competitivas o líderes, pero también por contribuir a la sustentabilidad (Salgado Beltrán et al., 2019; Salas Álvarez et al., 2020).

Esta sustentabilidad debe extenderse a la innovación, la cual tiene que ver con mejoras en las técnicas o métodos de producción o nuevas formas de comercialización (Velázquez-Juárez et al., 2016). Asimismo, tiene una función relevante porque contribuye al crecimiento de la riqueza (Grossman \& Helpman, 1993) vía el aprendizaje organizacional, que aumenta la competitividad, la productividad y la calidad de los bienes y servicios (Porter, 1982; Urarte, 2015; Londoño-Patiño \& Acevedo-Álvarez, 2018).

En este marco, las principales organizaciones generadoras de innovación son las empresas. Estas últimas para crecer requieren aplicar nuevos conocimientos científicos, comprar insumos, contratar personal y adquirir nuevas tecnologías que les permitan posicionarse y adaptarse a los cambios del mercado y responder a la sustentabilidad (Kuzma et al., 2020). Para innovar, las empresas necesitan colaborar con otras empresas, establecer relaciones con universidades y centros tecnológicos e incluso requieren del apoyo del gobierno. Una forma de estudiar las configuraciones entre los actores antes citados son las redes del conocimiento (Lee \& Miozzo, 2019; García Garnica, 2019).

En el esquema de redes, quienes participan en el proceso de innovación intercambian saberes, nuevas prácticas, experiencias y diferentes tipos de recursos a partir de acuerdos informales o formales (Trujillo Dávila et al., 2007; van Geenhuize \& Ye, 2014). Estas redes para funcionar se basan principalmente en la confianza, la autonomía y la autorregulación.

Un esquema adicional que estudia las relaciones de cooperación y competencia entre los diferentes actores que se vinculan en el ambiente económico-social son los ecosistemas de innovación o de negocios (Bacon et al., 2020). Esta propuesta enfatiza la co-evolución de las redes informales y los acuerdos formales entre empresas, universidades, el Gobierno y cualquier otra entidad. Lo importante son las capacidades que las organizaciones pueden adquirir de su entorno, a partir de intercambiar, aplicar y acumular conocimientos, habilidades y recursos ligados a la innovación (Möller \& Halinen, 2017; De Vasconcelos Gomes et al., 2018).

Por otro lado, teniendo en cuenta la ubicación geográfica, también se ha analizado la conformación de redes de colaboración bajo el concepto de cluster. Este concepto implica analizar los diferentes tipos de interconexiones que se observan entre las empresas, sus proveedores especializados, los clientes y las instituciones de Gobierno en una región determinada (Porter, 1999b). Bajo el modelo de análisis orientado por cluster, las empresas buscan estrategias de competitividad que les permitan incrementar el valor asociado a la cadena de suministro en la que se ubican. Por cadena de suministro se entiende «una combinación de procesos, funciones, actividades, relaciones y vías por las que los productos, los servicios, la información y las transacciones financieras se desplazan dentro de y entre 
Indicadores de sustentabilidad en empresas manufactureras y vinculación con instituciones generadoras de conocimiento: estudio de caso

las empresas a partir del fabricante original hasta el usuario o consumidor final» (Gattorna, 2006, p. 40). Es decir, se organizan en redes.

Al pertenecer a este tipo de redes, las empresas pueden reducir costos de logística, intercambiar bienes y servicios, conseguir información y conocimientos especializados, compartir gastos de infraestructura, contratar personal calificado y obtener los insumos que favorezcan la innovación (Porter, 1999; Delgado et al., 2016). Es necesario que como resultado de la interacción social del cluster se genere un valor compartido con la comunidad (Suire \& Vicente, 2009). Las empresas deben considerar su ventaja competitiva pero también diseñar una estrategia que contemple beneficios sociales ligados al uso de la energía y el agua, la creación de empleos, los programas de bienestar tanto para los empleados como para sus familias y la evaluación del impacto ambiental (Porter \& Kramer, 2011; Knight et al., 2020).

Asimismo, otros estudios enfatizan el papel de las redes en tanto son la base de los vínculos organizacionales e institucionales que se dan en la estructura económico-social, el comportamiento de los actores involucrados, así como los factores que facilitan la innovación, el incremento de la productividad y la sustentabilidad (Gándara Mendoza \& Villafán Vidales, 2019). En este marco, la posibilidad de que las empresas sean más competitivas y hagan uso de sus recursos eficientemente no puede hacerse de manera aislada y al margen de considerar los impactos ambientales (Riesener et al., 2019).

En el mismo sentido, Seuring y Muller (2008) señalan que las empresas deben aspirar a conformar cadenas de suministro sustentables, es decir, crear redes de cooperación en las que los involucrados consideren aspectos económicos, sociales y ambientales. Al respecto, Chardine-Baumann y BottaGenoulaz (2014) han identificado 66 indicadores ligados a las prácticas empresariales a nivel de su contribución económica, su desempeño ambiental y la responsabilidad social. Esta propuesta se desglosa más adelante, en la parte metodológica de este documento, ya que el referente empírico que se reporta se pregunta sobre estos indicadores.

De acuerdo con la OECD (2010), las universidades son actores importantes como productoras de conocimientos científicos, los cuales se usan para resolver problemas tecnológicos, sociales o económicos. Algunas empresas han logrado mejorar sus procesos de innovación al establecer fuertes vínculos formales e informales (a mediano y largo plazo) con las universidades (Díez Vial \& Montoro Sánchez, 2016). Sin embargo, para Fernández de Lucio et al., (2000), el tipo de vínculos que hay entre las universidades y las empresas puede ser fuerte o débil. Dicha relación depende del tamaño de las organizaciones, el tipo de actividad económica en la que se ubiquen, la calidad técnica y profesional de los recursos humanos involucrados, así como de la actitud que las empresas tengan respecto a la innovación.

Dese el punto de vista de Pérez Esparza (2016), la relación que tienen las universidades con las empresas puede identificarse a partir de dos variables: las fortalezas y obstáculos para implementar programas de vinculación, y los incentivos para promover las actividades de colaboración con las instituciones educativas. Entre algunas de las dificultades que tienen las empresas para acercarse a las universidades, se encuentra que los centros educativos no conocen la realidad que enfrenta la industria, el conocimiento que generan las universidades no se da a conocer a las empresas, los principales vínculos se dan a través de las prácticas profesionales, las instalaciones universitarias muchas veces están limitadas y los investigadores tienen poco interés por acercarse a lo que sucede en las organizaciones. 
Para Alvarado-Borrego (2009), es necesario fortalecer las relaciones entre las empresas y las universidades a partir de impulsar la confianza y el diálogo, crear fuentes de información que den cuenta de las necesidades que tienen las empresas y de los recursos profesionales y los conocimientos que pueden ofrecer las universidades, establecer líneas de investigación sobre la base de diagnósticos y mejoras socioeconómicas y tecnológicas previas, y mejorar el sistema de prácticas profesionales.

De igual forma, Sarabia-Altamirano (2016) y García-Pérez-de-Lema et al., (2017) consideran que las relaciones entre las universidades y las empresas favorecen el desarrollo de las naciones en la medida que se intercambian conocimientos y se generan tecnologías e innovaciones que favorecen a la sociedad. Estos vínculos pueden ser formales e informales y pueden mejorarse a partir del impulso de políticas públicas que apoyen con recursos y estímulos dicha relación. La base de este vínculo puede darse a partir de asesorías o consultorías, patentes, proyectos conjuntos de colaboración y la formación de recursos humanos, las cuales contribuyan a la transferencia de conocimientos y tecnologías enfocadas a la solución de problemas específicos.

Vega Jurado et al., (2011) concuerdan en que la innovación se incrementa en aquellas condiciones en las que se estrechan los lazos de cooperación entre las universidades y las empresas. Sin embargo, plantean que es importante impulsar políticas públicas que fortalezcan las capacidades de investigación de las universidades y de programas que promuevan la inserción de los profesionistas en el mercado laboral; por tanto, el Gobierno es un factor clave que facilita la colaboración universidad y empresa.

Por lo antes expuesto, desde la universidad pública estatal se reconoce que el mundo actual se enmarca en un contexto globalizado donde se presentan retos en distintos rubros, económico, productivo, ambiental, y, por ende, en el educativo (Giddens, 1990). En este sentido, la formación universitaria - tanto los profesionistas como de investigadores-, en consonancia con una sociedad en permanente cambio, necesitan ser actualizados para transitar hacia nuevos horizontes que incluyan como eje fundamental la innovación.

La universidad necesita mantener e incrementar la calidad como un componente transversal que responda tanto a la formación de ciudadanos críticos como a las demandas del mercado laboral, y que le permita egresar estudiantes con conocimientos académicos articulados con las habilidades necesarias para su desempeño profesional de excelencia (Universidad Autónoma del Estado de Morelos [UAEM], 2019, p. 5).

Los desafíos son muy numerosos, pero corresponde a las diversas áreas de conocimiento en las universidades y de otras instituciones generadoras de conocimiento preparar a los jóvenes que habrán de ingresar a las opciones laborales en el marco de exigencias, entre otras las de la Industria 4.0 (Imkamp, et al., 2016), innovar las condiciones e imaginar las propuestas de formación profesional y de vinculación para quienes seguirán trabajando e investigando en los diversos campos.

De ahí que estudiar la relación entre vinculación con IGC y sustentabilidad en las cadenas de suministro como parte de la formación de recursos humanos es un compromiso de las actividades que se tienen que realizar en las instituciones educativas y de investigación, para conocer las necesidades de desarrollo de tecnología que requiere el sector productivo. 
El objetivo general de este trabajo es correlacionar la implementación de indicadores de sustentabilidad en las cadenas de suministro, a las que pertenecen las medianas y grandes empresas manufactureras ubicadas en Morelos, con el nivel de vinculación que estas firmas tienen con las universidades y los centros de investigación en dicho estado. La hipótesis de trabajo es que existe una relación estadísticamente significativa entre la vinculación académica y la implementación de indicadores de sustentabilidad.

\section{METODOLOGÍA}

La metodología empleada en esta investigación, desde un enfoque cuantitativo, prevé un alcance descriptivo y correlacional: toma la encuesta como instrumento de acopio utilizada en la investigación de negocios y logística (Halldorsson \& Arlbjørn, 2005). A saber, la encuesta es considerada un método de investigación en sí mismo (Quivy \& Campenhoudt, 2017).

El diseño de la investigación propuso hacer una correlación no paramétrica de Rho de Spearman (Reguant-Álvarez et al., 2018) entre las variables «Indicadores de Sustentabilidad», «Vinculación UAEM» y «Vinculación Otras» con el objeto de comprobar la hipótesis. Se utilizó dicha correlación, dado que la muestra es pequeña y no se puede comprobar que se cuenta con una distribución normal (Mondragón-Barrera, 2014). Para el análisis de los datos, se utilizó el programa IBM SPSS Stadistics Versión 21. Los resultados obtenidos se muestran en un apartado más adelante.

La encuesta se construyó con base en el trabajo de Chardine-Baumann y Botta-Genoulaz (2014), que propone 66 indicadores en las áreas de desempeño ambiental, responsabilidad social y contribución económica. Los autores mencionados utilizaron estos indicadores para crear un modelo matemático que pueda evaluar la sustentabilidad en las cadenas de suministro. El trabajo que se está reportando transforma los indicadores de Chardine-Baumann y Botta-Genoulaz en preguntas que forman parte de la encuesta. Las respuestas a estas preguntas se contestaron en escala Likert (Matas, 2018), con opciones de respuesta donde 0 No aplica nunca en ningún área de la empresa, 1 Aplica algunas veces en algunas áreas de la empresa, 2 Aplica siempre en algunas áreas de la empresa y 3 Aplica siempre para toda la empresa. Las dimensiones y categorías se muestran en la tabla 1. Se hizo un pilotaje con un grupo de pasantes de ingeniería y los resultados obtenidos muestran que el instrumento es válido y confiable, dado que su Alfa de Cronbach fue de 0.957 y el análisis factorial arroja un solo factor con un KMO de 0.448 y un nivel de significancia de 0.05 .

La encuesta se complementó con dos preguntas dicotómicas relacionadas con la vinculación existente entre las empresas y las instituciones de educación superior y de investigación, particularmente con la UAEM, institución establecida en el mismo estado en el que se encuentran las empresas. La primera pregunta indagó particularmente la vinculación con la UAEM y la segunda, con otras instituciones de educación superior o de investigación diferentes a la UAEM. Las formas de respuesta a estas preguntas son Sí, para cuando existe vinculación, y NO, para cuando no. Se estableció una tercera pregunta dividida en actividades que realizan las empresas con las instituciones de educación superior o de investigación: prácticas profesionales, proyectos de investigación y desarrollo, cursos y diplomados, pláticas de divulgación, asesoramiento y consultoría y servicios especializados, cuya respuesta consistía únicamente en marcar aquellas que se llevaran a cabo. 
Tabla 1. Caracterización del modelo de desempeño sustentable

Table 1. Characterization of the sustainable performance model

\begin{tabular}{|c|c|c|c|}
\hline Dimensión & Campo & $\begin{array}{l}\text { Número de } \\
\text { Subcampos }\end{array}$ & Subcampos \\
\hline \multirow{5}{*}{ Económica } & Confiabilidad & 4 & $\begin{array}{l}\text { Servicio al cliente, servicio de los proveedores, } \\
\text { confiabilidad de los inventarios, confiabilidad de los } \\
\text { pronósticos }\end{array}$ \\
\hline & Capacidad de respuesta & 8 & $\begin{array}{l}\text { Del diseño, de la compra, del proveedor, de la } \\
\text { producción, de la entrega, de la venta, de los } \\
\text { retornos, de la cadena de suministro }\end{array}$ \\
\hline & Flexibilidad & 4 & $\begin{array}{l}\text { De los proveedores, de la oferta, de la producción, de } \\
\text { la entrega }\end{array}$ \\
\hline & Rendimiento financiero & 7 & $\begin{array}{l}\text { Costos de diseño, costos de compra, costos de origen, } \\
\text { costos de producción, costos de entrega, costos de } \\
\text { retorno, costo de la cadena de suministro }\end{array}$ \\
\hline & Calidad & 3 & $\begin{array}{l}\text { Calidad del producto o servicio, calidad del } \\
\text { desempeño de los proveedores, calidad en la } \\
\text { producción }\end{array}$ \\
\hline \multirow{5}{*}{ Ambiental } & Administración Ambiental & 4 & $\begin{array}{l}\text { Presupuesto ambiental, certificación ambiental, } \\
\text { cumplimiento ambiental, implicaciones con los } \\
\text { trabajadores }\end{array}$ \\
\hline & Uso de recursos & 5 & $\begin{array}{l}\text { Energía renovable, agua reciclada, insumos derivados } \\
\text { del reciclaje, salidas reciclables, residuos reciclables }\end{array}$ \\
\hline & Contaminación & 4 & $\begin{array}{l}\text { Contaminación del aire, contaminación del agua, } \\
\text { contaminación del suelo, otros tipos de } \\
\text { contaminación }\end{array}$ \\
\hline & Peligrosidad & 3 & $\begin{array}{l}\text { Entradas peligrosas, salidas peligrosas, desechos } \\
\text { peligrosos }\end{array}$ \\
\hline & Entorno natural & 4 & $\begin{array}{l}\text { Servicios eco sistémicos, respeto a la biodiversidad, } \\
\text { uso de suelo, desarrollo de zonas urbanas y rurales }\end{array}$ \\
\hline \multirow{5}{*}{ Social } & Condiciones de trabajo & 5 & $\begin{array}{l}\text { Desarrollo del empleo, condiciones de trabajo, } \\
\text { respeto al dialogo social, salud y seguridad, desarrollo } \\
\text { de los recursos humanos }\end{array}$ \\
\hline & Derechos humanos & 3 & $\begin{array}{l}\text { Trabajo forzoso e infantil, libertad de asociación, } \\
\text { discriminación }\end{array}$ \\
\hline & Compromiso con la sociedad & 5 & $\begin{array}{l}\text { Implicación con la comunidad local, educación, } \\
\text { cultura y desarrollo tecnológico, creación de empleo, } \\
\text { asistencia sanitaria, inversión social }\end{array}$ \\
\hline & Problemas de los clientes & 4 & $\begin{array}{l}\text { Mercadotecnia e información, salud y seguridad, } \\
\text { protección de datos confidenciales, acceso a servicios } \\
\text { esenciales }\end{array}$ \\
\hline & Prácticas de negocios & 3 & $\begin{array}{l}\text { Lucha contra la corrupción, comercio justo, } \\
\text { promoción de la responsabilidad social corporativa } \\
\text { en su ámbito de influencia }\end{array}$ \\
\hline
\end{tabular}

Fuente: Chardine-Baumann y Botta-Genoulaz (2014, p. 141).

Las empresas seleccionadas para responder a dicha encuesta se obtuvieron de la base de datos del Directorio Estadístico Nacional de Unidades Económicas, Denue (Instituto Nacional de Estadística y Geografía [Inegi], 2018). Esta base de datos ofrece identificación, ubicación, actividad económica y tamaño de más de cinco millones de unidades económicas activas en el territorio mexicano. Se 
seleccionaron aquellas empresas de más de 30 trabajadores, ya que, de acuerdo a la estratificación establecida, estas empresas se consideran como medianas y grandes (Instituto Nacional de Estadística y Geografía [Inegi], 2015). Se seleccionó como actividad económica a las industrias manufactureras, porque son las que forman parte de cadenas de suministro más largas y complejas. En cuanto a la ubicación geográfica, se seleccionó al estado de Morelos; con fines comparativos, se preguntó por la vinculación con la universidad pública autónoma de esa entidad, la UAEM y con «Otras» instituciones generadoras de conocimiento.

Se estableció contacto con las empresas de manera telefónica y por correo electrónico y se reunió a los encargados de las actividades relacionadas con su sustentabilidad para explicarles los fines académicos de la misma, se les solicitó su consentimiento para el uso de los datos y se les aplicó la encuesta. La decisión de concertarlos obedece a la experiencia con trabajos anteriores realizados por el mismo grupo de investigación, en los cuales se encontró que la tasa de respuesta para la aplicación y el tiempo empleado en el trabajo de campo eran más eficientes si se reunía en un solo evento a los directivos de los cuales se quiere obtener información. Acudieron representantes de 36 empresas, de las cuales se darán los resultados en el apartado correspondiente.

\section{RESULTADOS}

Las empresas encuestadas corresponden a 26 diferentes actividades económicas, entre las que destacan la fabricación de cosméticos, perfumes y otras preparaciones de tocador, y el mantenimiento industrial. En cuanto al tamaño de las empresas, en la tabla 2 se presentan los resultados, en los que se enfatiza que el $33 \%$ de ellas son empresas grandes, con más de 250 trabajadores.

Tabla 2. Tamaño de las empresas encuestadas

Table 2. Size of the companies surveyed

\begin{tabular}{|c|c|c|c|c|c|}
\hline \multicolumn{6}{|c|}{ Estrato de personal } \\
\hline & & Frecuencia & Porcentaje & Porcentaje válido & $\begin{array}{l}\text { Porcentaje } \\
\text { acumulado }\end{array}$ \\
\hline \multirow{5}{*}{ Válidos } & $31-50$ personas & 10 & 27.8 & 27.8 & 27.8 \\
\hline & 51-100 personas & 7 & 19.4 & 19.4 & 47.2 \\
\hline & $101-250$ personas & 7 & 19.4 & 194 & 66.7 \\
\hline & 251 y más personas & 12 & 33.3 & 33.3 & 100.0 \\
\hline & Total & 36 & 100.0 & 100.0 & \\
\hline
\end{tabular}

Fuente: elaboración propia, datos obtenidos con el software IBM SPSS Stadistics V. 21.

Los resultados referentes a los indicadores de sustentabilidad se ciñen al modelo de desempeño sustentable de Chardine-Baumann y Botta-Genoulaz (2014), mostrado en la tabla 1 y a los tipos de respuesta de acuerdo con la escala Likert establecida en la metodología:

0 No aplica nunca en ningún área de la empresa,

1 Aplica algunas veces en algunas áreas de la empresa,

2 Aplica siempre en algunas áreas de la empresa y 
3 Aplica siempre para toda la empresa.

De acuerdo a la tabla 3 y los tipos de respuesta, las empresas encuestadas aplican los indicadores de sustentabilidad como un todo «siempre en algunas áreas de la empresa». En cuanto a la aplicación de los indicadores económicos, también lo hacen «siempre en algunas áreas de la empresa». De las tres dimensiones, la aplicación de los indicadores ambientales es la que arroja el valor más bajo en la media, traduciéndose en la respuesta «algunas veces en algunas áreas de la empresa». A pesar de lo que suponía inicialmente el grupo de trabajo, la aplicación de los indicadores sociales tuvo una respuesta con la media más alta, que significa que la aplicación de estos indicadores se hace «siempre para toda la empresa».

Tabla 3. Descriptivos estadísticos de las dimensiones del modelo de desempeño sustentable Table 3. Descriptive statistics of the dimensions of the sustainable performance model

\begin{tabular}{cccc}
\hline \multicolumn{4}{c}{ Estadísticos descriptivos } \\
\hline $\begin{array}{c}\text { Indicadores de } \\
\text { Sustentabilidad }\end{array}$ & 36 & 1.9946 & 0.50829 \\
\hline Dimensión Económica & 36 & 2.0495 & 0.66358 \\
\hline Dimensión Ambiental & 36 & 1.2075 & 0.57127 \\
\hline Dimensión Social & 36 & 2.7269 & 0.59439 \\
\hline N válido (según lista) & 36 & & \\
\hline
\end{tabular}

Fuente: elaboración propia, datos obtenidos con el software IBM SPSS Stadistics V. 21.

En lo referente a la vinculación, los resultados estadísticos dan una media de 0.5 para la UAEM y 0.47 para otras instituciones. Estas cifras muestran que solo la mitad de las empresas encuestadas tienen algún tipo de colaboración con la Universidad Autónoma del Estado de Morelos o con otras instituciones afines.

Los resultados que se obtuvieron en la encuesta en cuanto a la correlación existente entre los indicadores de sustentabilidad y la vinculación que guardan las empresas con las instituciones de educación superior o centros de investigación es explicada a continuación.

La correlación se hizo primeramente entre dos variables: «Vinculación UAEM» e «Indicadores de Sustentabilidad». La primera es una variable dicotómica, la cual solo fue contestada por los encuestados como sí o no. La segunda es el promedio de las dimensiones económica, social y ambiental derivadas de los 66 indicadores que se propusieron para la evaluación de la sustentabilidad en las cadenas de suministro. El tipo de correlación usado fue el Rho de Spearman, dado que la muestra es pequeña (36 empresas) y no se puede comprobar que se cuenta con una distribución normal. El resultado obtenido para las dos variables antes citadas se muestra en la tabla 4. 
Indicadores de sustentabilidad en empresas manufactureras y vinculación con instituciones generadoras de conocimiento: estudio de caso

Tabla 4. Correlación Rho de Spearman entre Vinculación UAEM e Indicadores de Sustentabilidad Table 4. Correlation (Spearman's rho) between Links with UAEM and Sustainability Indicators

\begin{tabular}{|c|c|c|c|c|}
\hline & & & $\begin{array}{l}\text { Vinculación } \\
\text { uaem }\end{array}$ & $\begin{array}{r}\text { Prácticas de } \\
\text { Sustentabilidad }\end{array}$ \\
\hline \multirow{6}{*}{ Rho de Spearman- } & \multirow{3}{*}{ Vinculación uaem } & $\begin{array}{l}\text { Coeficiente de } \\
\text { correlación }\end{array}$ & 1.000 & 0.037 \\
\hline & & Sig. (bilateral) & . & 0.828 \\
\hline & & $\mathrm{N}$ & 36 & 36 \\
\hline & \multirow{3}{*}{$\begin{array}{l}\text { Indicadores de } \\
\text { Sustentabilidad }\end{array}$} & $\begin{array}{l}\text { Coeficiente de } \\
\text { correlación }\end{array}$ & 0.037 & 1.000 \\
\hline & & Sig. (bilateral) & 0.828 & . \\
\hline & & $\mathrm{N}$ & 36 & 36 \\
\hline
\end{tabular}

Fuente: elaboración propia, datos obtenidos con el software IBM SPSS Stadistics V. 21.

Como se puede observar, la correlación existente de 0.037 es una correlación positiva muy débil (Hernández-Sampieri \& Mendoza-Torres, 2018). Esto indica que, para la implementación de los indicadores de sustentabilidad en las cadenas de suministro de las empresas encuestadas, no es relevante la vinculación con la UAEM.

En el mismo tenor, se les preguntó a las empresas si tenían convenio de vinculación con otra institución de educación superior o de investigación diferente a la UAEM. Las respuestas también fueron dicotómicas: sí y no. A esta variable se le nombró «Vinculación Otras» y se correlacionó con la variable «Indicadores de Sustentabilidad». Los resultados se muestran en la tabla 5.

Tabla 5. Correlación Rho de Spearman entre Vinculación Otras e Indicadores de Sustentabilidad

Table 5. Correlation (Spearman's rho) between Links with Other Institutions and Sustainability Indicators

\begin{tabular}{|c|c|c|c|c|}
\hline & & & $\begin{array}{c}\text { Prácticas de } \\
\text { Sustentabilidad }\end{array}$ & Vinculación Otras \\
\hline \multirow{6}{*}{ Rho de Spearman } & \multirow{3}{*}{$\begin{array}{l}\text { Indicadores de } \\
\text { Sustentabilidad }\end{array}$} & $\begin{array}{c}\text { Coeficiente de } \\
\text { correlación }\end{array}$ & 1.000 & $0.453^{* *}$ \\
\hline & & Sig. (bilateral) & & 0.006 \\
\hline & & $N$ & 36 & 36 \\
\hline & \multirow{3}{*}{ Vinculación Otras } & $\begin{array}{c}\text { Coeficiente de } \\
\text { correlación }\end{array}$ & $0.453^{* *}$ & 1.000 \\
\hline & & Sig. (bilateral) & 0.006 & . \\
\hline & & $\mathrm{N}$ & 36 & 36 \\
\hline
\end{tabular}

** La correlación es significativa al nivel 0,01 (bilateral).

Fuente: elaboración propia, datos obtenidos con el software IBM SPSS Stadistics V. 21.

El coeficiente de correlación de 0.453 muestra que existe una correlación positiva media con un nivel de significancia de 0.01. Esto revela que, para la implementación de los indicadores de sustentabilidad en las cadenas de suministro de las empresas encuestadas, es medianamente relevante la vinculación con instituciones de educación superior o de investigación diferentes a la UAEM. 
Cuando se conjunta tanto la relación con la UAEM con otras instituciones (a través de la suma de seis actividades de vinculación: prácticas profesionales, proyectos I+D, cursos y diplomados, pláticas de divulgación, asesoramiento y consulta y servicios especializados) surgen los resultados estadísticos que se muestran en la tabla 6 .

Tabla 6. Correlación Rho de Spearman entre Actividades de Vinculación e Indicadores de Sustentabilidad Table 6. Correlation (Spearman's rho) between Linkage Activities and Sustainability Indicators

\begin{tabular}{|c|c|c|c|c|}
\hline & & & $\begin{array}{c}\text { Prácticas de } \\
\text { Sustentabilidad }\end{array}$ & $\begin{array}{c}\text { Actividades de } \\
\text { Vinculación }\end{array}$ \\
\hline \multirow{6}{*}{ Rho de Spearman } & \multirow{3}{*}{ Indicadores de Sustentabilidad } & Coeficiente de correlación & 1.000 & -0.011 \\
\hline & & Sig. (bilateral) & . & 0.951 \\
\hline & & $\mathrm{N}$ & 36 & 36 \\
\hline & \multirow{3}{*}{ Actividades de Vinculación } & Coeficiente de correlación & -0.011 & 1.000 \\
\hline & & Sig. (bilateral) & 0.951 & . \\
\hline & & $\mathrm{N}$ & 36 & 36 \\
\hline
\end{tabular}

Fuente: elaboración propia, datos obtenidos con el software IBM SPSS Stadistics V. 21.

El resultado -0.011 es una correlación negativa extremadamente débil que se puede interpretar como nula, lo que muestra que las actividades de vinculación no tienen relación alguna con la implementación de indicadores de sustentabilidad en las cadenas de suministro de las empresas encuestadas.

Los resultados encontrados no son alentadores en cuanto a la utilidad de la vinculación con respecto a la sustentabilidad de las cadenas de suministro del presente caso. Un análisis detallado de lo anterior se presenta en el apartado siguiente.

\section{DISCUSIÓN}

El objetivo general de la investigación es correlacionar la implementación de indicadores de sustentabilidad en las cadenas de suministro a las que pertenecen las medianas y grandes empresas manufactureras del estado de Morelos con la vinculación con universidades y centros de investigación establecidas en el mismo estado. La hipótesis planteada en esta investigación se acepta con base en los resultados de la encuesta aplicada, los cuales confirman que existe una relación estadísticamente significativa, débil respecto a la universidad pública de la entidad y media respecto a otras instituciones entre la vinculación y la implementación de los indicadores de sustentabilidad.

Se encontró que la relación estadística muestra coincidencia con lo expresado por Fernández de Lucio et al., (2000), en cuanto a que los vínculos entre las universidades, IGC en este trabajo, y las empresas pueden ser fuertes o débiles. Particularmente, en el caso bajo estudio, para la implementación de los indicadores de sustentabilidad en las cadenas de suministro de las empresas encuestadas, la vinculación con la UAEM no es relevante, es decir, las empresas pueden prescindir de los conocimientos que esta genera. Esto sugiere que los conocimientos que se formulan en esta institución no cumplen las expectativas o necesidades de las empresas. Pese a que existe una relación de colaboración con ellas, y que estas actividades se refieren a prácticas profesionales, pláticas de divulgación, proyectos de investigación y desarrollo, cursos y diplomados, asesoramiento y 
Indicadores de sustentabilidad en empresas manufactureras y vinculación con instituciones generadoras de conocimiento: estudio de caso

consultoría y servicios especializados, de estas, la primera está relacionada con complementar la formación del profesional, mientras que las otras muestran un sentido de aplicación del conocimiento entra ambas entidades (la universidad y la empresa). No obstante, se pretende que el conocimiento que se genera en las universidades y centros de investigación sea en consecuencia de las necesidades de las empresas. Esta condición no se cumple y deja en desventaja a la universidad pública mexicana, la cual se observa desfasada de los requerimientos de la 4a Revolución Industrial (Schwab, 2017); por tanto, convida a las empresas a depender de los conocimientos que se producen en el exterior del país.

El aporte de esta investigación estriba en que la existencia de convenios de colaboración es un elemento que hace visibles a las instituciones de educación superior (IES) y centros de investigación en las empresas; a su vez, esto se convierte en un elemento para la implementación de los indicadores de sustentabilidad en las cadenas de suministro de las empresas encuestadas. Sin embargo, los datos muestran que el establecimiento de estos convenios es una actividad con escaso impacto económico y tecnológico o de innovación, lo cual hace inferir que los beneficios por contar con estos acuerdos no constituyen una ventaja tangible que motive a las empresas a generar, promover o mantener este tipo de vinculación.

Al reflexionar sobre los dos párrafos previos, esta información indica que el esfuerzo de las IGC, primero por ser visibilizadas, $y$, después, por generar conocimientos y patentes y ser reconocidas por las empresas como entidades que aportan al desarrollo de las mismas, es el primer reto que deben enfrentar. Por tanto, la promoción de las actividades para establecer contacto con las empresas es una de las vetas que deja esta investigación y alerta de la necesidad de llevar a cabo acciones concretas y continuas para mantener e incrementar las relaciones de colaboración con las empresas. Ante la circunstancia antes descrita, cobra relevancia lo que declaran Vega Jurado et al., (2011) acerca de la importancia de impulsar políticas públicas para fortalecer las capacidades de investigación de las universidades, de tal manera que sean un referente para las empresas. De la misma forma, se acepta el papel del Gobierno como facilitador de la colaboración entre la universidad y empresa, justamente, haciendo vivo el modelo denominado de la triple hélice (Etzkowitz \& Leydesdorff, 2000), en el que existen sinergias entre el Estado, la empresa y las IGC, que son generadoras del conocimiento y contribuyan a la innovación de las organizaciones.

Para hacer realidad que la investigación de las universidades y de los centros de investigación estén al servicio de la sociedad, se coincide con Sarabia-Altamirano (2016) en que, como lo menciona supra, los vínculos con la empresa pueden ser formales e informales y, por tanto, lograr una relación que se base en la transferencia de conocimientos y tecnologías enfocadas a la solución de problemas específicos. A su vez, esto provoca la innovación y en consecuencia el desarrollo de las naciones.

Una alternativa que emerge de esta investigación para potenciar la vinculación universidad-empresa se encuentra en el apartado teórico de este artículo. Son los denominados cluster, ya que el beneficio que se pretende no es solo para la empresa, sino también para la comunidad, en especial aquellos relacionados con la consecución de empleos dignos y con procesos productivos que se ocupen de prevenir y resarcir el daño ocasionado al medio ambiente.

Por supuesto que las reflexiones en torno a los hallazgos indican que hay una ética de la sustentabilidad (Galano et al., 2002) en la hipótesis de este trabajo. Por una parte, es menester que tanto las empresas como las instituciones de educación superior y los centros de investigación generen principios éticos y actúen en consecuencia para realmente mitigar los efectos del cambio 
climático y realizar acciones orientadas a asegurar que se satisfagan las necesidades del presente, sin comprometer las necesidades de futuras generaciones. Es decir, hacer de la sustentabilidad un quehacer de todos.

En síntesis, la vinculación es una tarea que compete tanto a la IES y centros de investigación como a las empresas, ya que ambas instancias interactúan en una sociedad donde el riesgo está presente desde finales del siglo XX. En este sentido, se ha señalado que:

La prognosis mediante cálculos de modelo de problemas en el mercado laboral tiene un efecto inmediato sobre la actitud educativa; la anticipación del desempleo inminente es una determinante esencial de la situación vital y del estado de ánimo del presente; la prognosis de la destrucción del medio ambiente y la amenaza atómica intranquilizan a una sociedad y son capaces de sacar a la calle a grandes partes de la generación joven. (Beck, 1998).

Es ahí donde la empresa, en el propio sentido de sobrevivir, muestra la responsabilidad social que le corresponde cualquiera que sea su ámbito de influencia.

\section{CONCLUSIONES}

La sociedad actual está atravesando por un conjunto de cambios económicos, políticos y tecnológicos. La globalización, el desarrollo de las nuevas tecnologías, la modificación en los gustos de los consumidores, la crisis socioambiental, los flujos en las migraciones y la inestabilidad financiera son algunos factores que están causando incertidumbre social. Estos fenómenos afectan a las empresas, las universidades, los centros tecnológicos e incluso al Gobierno. Particularmente, queda claro que ningún actor social puede adaptarse al nuevo contexto de manera aislada; se requiere de la cooperación de todos para incrementar la riqueza económica, mejorar la distribución del ingreso, satisfacer las necesidades sociales y enfrentar el cambio climático y sus consecuencias. A saber, se requiere de la colaboración de todos para lograr un desarrollo sustentable.

Esta investigación enfatiza precisamente que una de las áreas de oportunidad, para el país de México, y particularmente para el caso de Morelos, está ligada a fortalecer las relaciones de cooperación entre las universidades y las empresas. Ante las necesidades que, en Morelos y en otros estados, tienen las empresas de mejorar sus productos, procesos y servicios de forma sustentable, sin duda la colaboración inter e intra empresa es fundamental. Pero también lo es el aprovechamiento de las oportunidades que potencialmente ofrecen la UAEM y otras instituciones afines en términos de fortalecer una colaboración basada en recursos y en conocimientos científicos.

Particularmente, y como resultado de la encuesta que se aplicó a empresas establecidas en este estado con el fin de indagar el nivel de colaboración que tienen con la UAEM, se muestra que, si bien hay acuerdos formales entre estas, en la práctica dicha vinculación es escasa. Esto se infiere a partir de analizar los indicadores de colaboración asociados a las prácticas profesionales, proyectos I+D, cursos y diplomados, pláticas de divulgación, asesoramiento y consulta y servicios especializados.

La UAEM tiene un enorme potencial en términos de líneas de generación y aplicación innovadora de conocimientos, a las cuales está adscrito un gran número de investigadores y profesores con vasta experiencia profesional. Además, cuenta con una amplia infraestructura y bases de datos, que son una inmensa fuente de información. Así mismo, cada año egresan muchos profesionistas que constituyen un importante capital humano para las empresas. 
Lo importante es crear las condiciones para que en el corto y mediano plazo se fortalezcan las relaciones entre las empresas, la universidad y el Gobierno en turno. No menos prioritario es que a futuro las investigaciones ligadas a analizar los vínculos entre las instituciones educativas y las empresas se amplíen en la región central del país, de tal manera que se coadyuve a la implementación de los indicadores de sustentabilidad.

\section{AGRADECIMIENTOS}

El financiamiento para este proyecto fue otorgado por el Programa para el Desarrollo Profesional Docente Prodep, Apoyo a la Reincorporación de Exbecarios, Carta de liberación DSA/103.5/16/10404 Folio UAEMOR-EXB-115.

\section{REFERENCIAS}

Alvarado-Borrego, A. (2009). Vinculación universidad-empresa y su contribución al desarrollo regional. Ra Ximhai, v. 5, n. 3, 407-414. URL

Bacon, E.; Williams, M. D.; Davies, G. (2020). Coopetition in innovation ecosystems: A comparative analysis of knowledge transfer configurations. Journal of Business Research, v. 115, 307-316. https://doi.org/10.1016/j.jbusres.2019.11.005

Beck, U. (1998). La sociedad del riesgo. Hacia una nueva modernidad. Paidós.

Chardine-Baumann, E.; Botta-Genoulaz, V. (2014). A framework for sustainable performance assessment of supply chain management practices. Computers \& Industrial Engineering, v. 76, 138-147. https://doi.org/10.1016/j.cie.2014.07.029

Delgado, M.; Porter, M. E.; Stern, S. (2016). Defining cluster of related industries. Journal of Economic Geography, v. 16, n. 1, 1-38. https://doi.org/10.1093/jeg/lbv017

De Vasconcelos Gomes, L. A.; Figuereido Facin, A. L.; Salerno, M. S.; Kazuo Ikenami, R. (2018). Unpacking the innovation ecosystem construct: Evolution, gaps and trends. Technological Forecasting and Social Change, v. 136, 30-48. https://doi.org/10.1016/j.techfore.2016.11.009

Díez Vial, I.; Montoro Sánchez, M. A. (2016). How knowledge links with universities may foster innovation: The case of a science park. Technovation, v. 50-51, 41-52. https://doi.org/10.1016/j.technovation.2015.09.001

Etzkowitz, H.; Leydesdorff, L. (2000). The dynamics of innovation: from National Systems and "Mode 2" to a Triple Helix of university-industry-government relations. Research Policy, v. 29, n. 2, 109-123. https://doi.org/10.1016/S0048-7333(99)00055-4 
Fernández de Lucio, I.; Castro Martínez, E.; Conesa Cegarra, F.; Gutiérrez Gracia, A. (2000). Las relaciones universidad-empresa entre la transferencia de resultados y el aprendizaje regional. Revista Espacios, v. 21, n. 2. URL

Fuster Morell, M.; Espelt, R. (2019). A Framework to Assess the Sustainability of Platform Economy: The Case of Barcelona Ecosystem. Sustainability, v. 11, n. 22, 6450. https://doi.org/10.3390/su11226450

Galano, C.; Curi, M.; Motomura, O.; Porto GonÁalves, C. W.; Silva, M.; Ángel, A.; Ángel, F.; Borrero, J. M.; Carrizosa, J.; Cortés, H.; Flórez, M.; Lozano, A.; Llano, A.; Mariño, J.; Mayr, J.; Schütze, K.; Valenzuela, L. C.; Mora, E.; Clark, I.; Elizalde, A.; Larraín, S.; Espinosa, M. F.; Haji Manchineri, S.; Franco, L. A.; Guerra, L. M.; Paredes, B.; Quadri, G.; Castro, G.; Tréllez, E.; Ramírez, J. C.; San Román, L.; Vilela, M.; Calderón, F.; Sánchez, R.; Leff, E. (2002). Manifiesto por la vida: por una ética para la sustentabilidad. Ambiente \& Sociedade, n. 10, 149-162. $\underline{U R L}$

Gándara Mendoza, A.; Villafán Vidales, K. B. (2019). Contribuciones de la teoría de redes hacia la sustentabilidad. Revista de la Facultad de Contaduría y Ciencias Administrativa, v. 4, n. 8, 114-129. URL

García Garnica, A. (2019). Pequeñas y Medianas Empresas (PyME) en el contexto de la innovación abierta. Una síntesis teórica. Teuken Bidikay, v. 10, n. 14, 101-120.

https://doi.org/10.33571/teuken.v10n14a5

García-Pérez-de-Lema, D.; Madrid-Guijarro, A.; Martin, D. P. (2017). Influence of university-firm governance on SMEs innovation and performance levels. Technological Forecasting and Social Change, v. 123, 250-261. https://doi.org/10.1016/j.techfore.2016.04.003

Gattorna, J. (2006). Supply chains are the business. Supply Chain Management Review, v. 6, n. 10, $42-49$.

Giddens, A. (1990). The consequences of Modernity. Polity Press.

Grossman, G. M.; Helpman, E. (1993). Innovation and growth in the global economy. The MIT Press.

Gutiérrez, T.; Hernández, J.; Carrillo, J. (2019). Desarrollo sustentable tecnológico: una aproximación teórica. Revista Ambientellanía, v. 2, n. 2, 11-19. URL

Hernández-Sampieri, R.; Mendoza-Torres, C. P. (2018). Metodología de la investigación. Las rutas cuantitativa, cualitativa y mixta. McGraw Hill Education.

Halldorsson, A.; Arlbjørn, J. S. (2005). Research methodologies in supply chain management: what do we know? In, Kotzab, H., Seuring, S., Muller, M. and Reiner, G. (eds.) Research Methodologies in Supply Chain Management. Berlin, Germany. Physica-Verlag, 107-122. https://doi.org/10.1007/3-7908-1636-1 
Imkamp, D.; Berthold, J.; Heizmann, M.; Kniel, K.; Manske, E.; Peterek, M.; Schmitt, R.; Seidler, J.; Sommer, K. D. (2016). Challenges and trends in manufacturing measurement technologythe "Industrie 4.0" concept. Journal of Sensors and Sensor Systems, v. 5, 325-335. https://doi.org/10.5194/jsss-5-325-2016

Instituto Nacional de Estadística y Geografía. (2015). Censos económicos 2014. Micro, pequeña, mediana y gran empresa: estratificación de los establecimientos. $\underline{\mathrm{URL}}$

Instituto Nacional de Estadística y Geografía. (2018). Directorio Estadístico Nacional de Unidades Económicas. $\underline{U R L}$

Knight, E.; Kumar, V.; Wójcik, D.; O'Neill, P. (2020). The competitive advantage of regions: Economic geography and strategic management intersections. Regional Studies, v. 54, n. 5, 591-595, https://doi.org/10.1080/00343404.2020.1739262

Kuzma, E.; Padilha, L. S.; Sehnem, S.; Julkovski, D. J.; Roman, D. J. (2020). The relationship between innovation and sustainability: A meta-analytic study. Journal of Cleaner Production, v. 259, 120745. https://doi.org/10.1016/j.jclepro.2020.120745

Lee H.; Miozzo, M. (2019). Which types of knowledge-intensive business services firms collaborate with universities for innovation? Research Policy, v. 48, n. 7, 1633-1646. https://doi.org/10.1016/j.respol.2019.03.014

Londoño-Patiño, J. A.; Acevedo-Álvarez, C. A. (2018). El aprendizaje organizacional (AO) y el desempeño empresarial bajo el enfoque de las capacidades dinámicas de aprendizaje. Revista CEA, v. 4, n. 7, 103-118. https://doi.org/10.22430/24223182.762

Matas, A. (2018). Diseño del formato de escalas tipo Likert: un estado de la cuestión. Revista Electrónica de Investigación Educativa, v. 20, n. 1, 38-47. https://doi.org/10.24320/redie.2018.20.1.1347

Möller, K.; Halinen, A. (2017). Managing business and innovation networks-From strategic nets to business fields and ecosystems. Industrial Marketing Management, v. 67, 5-22. https://doi.org/10.1016/j.indmarman.2017.09.018

Mondragón-Barrera, M. A. (2014). Uso de la correlación de Spearman en un estudio de intervención en fisioterapia. Movimiento científico, v. 8, n. 1, 98-104. https://doi.org/10.33881/2011-7191.mct.08111

OECD. (2010). Educación Hoy: la perspectiva de la OCDE. URL

Pérez Esparza, A. (2016). Razones que motivan la vinculación de la universidad con la empresa: Análisis comparativo México y Argentina. Revista RAITES, v. 2, n. 4, 10-28. URL

Porter, M. E. (1982). Estrategia competitiva. Compañía Editorial Continental.

Porter, M. E. (1999). Ser competitivo: nuevas aportaciones y conclusiones. Ediciones Deusto. 
Porter, M. E. (1999b). Los "clusters" y la nueva economía de competencia. Revista Palmas, v. 20, n. 4, 51-65. URL

Porter, M.; Kramer, M. R. (2011). Creating shared value. Harvard Business Review, v. 89, n. 1-2, 2-17.

Quivy, R.; Campenhoudt, L. (2017). Manual de investigación en ciencias sociales. Limusa.

Reguant-Álvarez, M.; Vilà-Baños, R.; Torrado-Fonseca, M. (2018). La relación entre dos variables según la escala de medición con SPSS. Revista d'Innovació y Recerca en Educació// REIRE, v. 11, n. $2,45-60 . \underline{U R L}$

Riesener, M.; Dölle C.; Khun M. (2019). Innovation ecosystem for industrial sustainability. Procedia CIRP, v. 80, 27-32. https://doi.org/10.1016/i.procir.2019.01.035

Salas Álvarez, W. T.; Medina León, A.; Medina Nogueira, D.; Ricardo Cabrera, H.; Salas Medina, P. E. (2020). Vínculo universidad-empresa para el desarrollo local sustentable en Pymes turísticas. Universidad y Sociedad, v. 12, n. 1, 350-358. URL

Salgado Beltrán, L.; Beltrán Morales, L. F.; García Moraga, R. E.; Beltrán Morales, F. A.; Subirá Lobera, M. E. (2019) Motivos para adoptar la sustentabilidad en los negocios. Agricultura Sociedad Y Desarrollo, v. 16, n. 3, 399-413. https://doi.org/10.22231/asyd.v16i3.1236

Sarabia-Altamirano, G. (2016). La vinculación universidad-empresa y sus canales de interacción desde la perspectiva de la academia, de la empresa y de las políticas públicas. CienciaUAT, v. 10, n. 2, 13-22. URL

Schrand, T. G. (2020) Utopianism and the equity path to sustainability. Journal of Environmental Studies and Sciences, 1-10. https://doi.org/10.1007/s13412-020-00619-4

Schwab, K. (2017). La cuarta revolución industrial. Debate.

Seuring S.; Muller M. (2008). From a Literature Review to a Conceptual Framework for Sustainable Supply Chain Management. Journal of Cleaner Production, v. 16, n. 15, 16991710. https://doi.org/10.1016/j.jclepro.2008.04.020

Suire, R.; Vicente, J. (2009). Why do some places succeed when others decline? A social interaction model of cluster viability. Journal of Economic Geography, v. 9, n. 3, 381-404. https://doi.org/10.1093/jeg/lbn053

Trujillo Dávila, M. A.; Guzmán Vásquez, A.; Becerra Plaza, G. E. (2007). Las redes organizacionales en la nueva forma de producción del conocimiento. Revista de la Escuela Colombiana de Ingeniería, n. 67, 81-92. URL

Universidad Autónoma del Estado de Morelos. (2019). Plan Institucional de Desarrollo (PIDE) 2018-2023. URL

Urarte, M. (2015). Estrategia, innovación y competitividad. Business Review, n. 243. URL 
van Geenhuizen, M.; Ye, Q. (2014). Responsible innovators: Open networks on the way to sustainability transitions. Technological Forecasting and Social Change, v. 87, n. C, 28-40. https://doi.org/10.1016/j.techfore.2014.06.001

Vega Jurado, J.; Manjarrés Henríquez, L.; Castro Martínez, E.; Fernández de Lucio, I. (2011). Las relaciones universidad-empresa: tendencias y desafíos en el marco del espacio iberoamericano del conocimiento. Revista Iberoamericana de Educación, v. 57, 109-124. https://doi.org/10.35362/rie570488

Velázquez-Juárez, J. A.; Valencia-Pérez, L. R.; Peña-Aguilar, J. M. (2016). El papel del modelo de la triple hélice como sistema de innovación para aumentar la rentabilidad en una Pyme comercializadora. Revista CEA, v. 2, n. 3, 101-112.

https://doi.org/10.22430/24223182.268 